\title{
Analysis of human MDM4 variants in papillary thyroid carcinomas reveals new potential markers of cancer properties
}

\author{
Andrea Prodosmo - Simona Giglio - Sonia Moretti • \\ Francesca Mancini - Flavia Barbi • Nicola Avenia • \\ Giusy Di Conza • Holger J. Schünemann • \\ Lorenza Pistola • Vienna Ludovini • Ada Sacchi • \\ Alfredo Pontecorvi • Efisio Puxeddu • Fabiola Moretti
}

Received: 12 June 2007 /Revised: 4 February 2008 / Accepted: 6 February 2008 /Published online: 12 March 2008

(C) The Author(s) 2008

\begin{abstract}
A wild-type (wt) p53 gene characterizes thyroid tumors, except for the rare anaplastic histotype. Because p53 inactivation is a prerequisite for tumor development, alterations of $\mathrm{p} 53$ regulators represent an alternative way to impair p53 function. Indeed, murine double minute 2 (MDM2), the main p53 negative regulator, is overexpressed in many tumor histotypes including those of the thyroid. A new p53 regulator, MDM4 (a.k.a. MDMX or HDMX) an analog of MDM2, represents a new oncogene although its impact on tumor properties remains largely unexplored. We estimated levels of MDM2, MDM4, and its variants, MDM4-S (originally HDMX-S) and MDM4-211 (originally HDMX211), in a group of 57 papillary thyroid carcinomas (PTC), characterized by wt tumor protein 53, in comparison to matched contra-lateral lobe normal tissue. Further, we evaluated the association between expression levels of these
\end{abstract}

A. Prodosmo · S. Giglio • F. Mancini • G. Di Conza • A. Sacchi

F. Moretti

Lab. Molecular Oncogenesis,

Regina Elena Cancer Institute,

Rome, Italy

\begin{abstract}
A. Prodosmo $\cdot$ S. Giglio $\cdot$ F. Mancini $\cdot$ A. Pontecorvi Inst. Medical Pathology, Catholic University,

Rome, Italy
\end{abstract}

S. Moretti $\cdot$ F. Barbi $\cdot$ E. Puxeddu

Dept. of Internal Medicine, University of Perugia,

Perugia, Italy

\section{F. Mancini · F. Moretti $(\bowtie)$}

Inst. of Neurobiology and Molecular Medicine,

National Council of Research,

Rome, Italy

e-mail: moretti@ifo.it

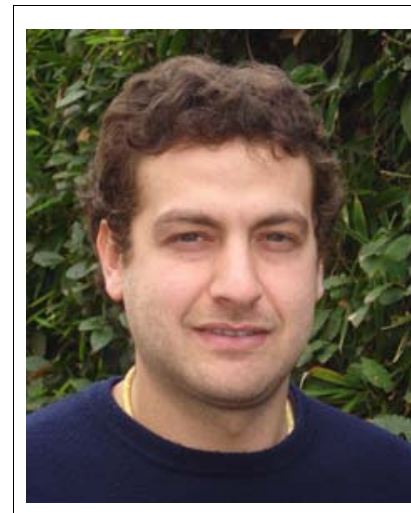

Andrea Prodosmo

received his degree in Molecular Biology from the University "La Sapienza" in Rome, Italy. He is presently a $\mathrm{Ph} . \mathrm{D}$. student in Endocrinology at the "Università Cattolica del Sacro Cuore" in Rome. His research interests include the study of the apoptotic pathways in response to genotoxic stress.

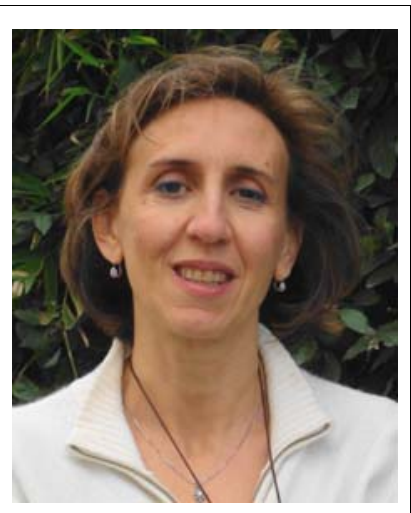

FABIOLA Moretti is presently a Group Leader at the Institute of Neurobiology and Molecular Medicine of National Council of Research, Rome, Italy. She received her doctoral training in Medical Genetics from University La Sapienza of Rome, Italy and her postdoctoral training in Basic Research in Oncology at the Leiden University Medical Center, The Netherlands. Her research interests include studies on the apoptotic response. genes and the histopathological features of tumors. Quantitative real-time polymerase chain reaction revealed a highly significant downregulation of MDM4 mRNA in tumor tissue compared to control tissue $(P<0.0001)$, a finding confirmed by western blot on a subset of 20 tissue pairs. Moreover, the tumor-to-normal ratio of MDM4 levels for each individual 
was significantly lower in late tumor stages, suggesting a specific downregulation of MDM4 expression with tumor progression. In comparison, MDM2 messenger RNA (mRNA) and protein levels were frequently upregulated with no correlation with MDM4 levels. Lastly, we frequently detected overexpression of MDM4-S mRNA and presence of the aberrant form, MDM4-211 in this tumor group. These findings indicate that MDM4 alterations are a frequent event in PTC. It is worthy to note that the significant downregulation of full-length MDM4 in PTC reveals a novel status of this factor in human cancer that counsels careful evaluation of its role in human tumorigenesis and of its potential as therapeutic target.

Keywords MDM4 · MDM2 - Thyroid carcinoma . MDM4 variants $\cdot \mathrm{p} 53$

\section{Introduction}

The murine double minute (MDM) family members are key regulators of levels and activity of the oncosuppressor p53 [1]. MDM2, acting as specific E3 ubiquitin ligase and as transcriptional repressor, is the best known among them. MDM2 overexpression has been observed in many human tumors characterized by tumor protein 53 (TP53) wild-type (wt) status, supporting the model of multiple means of p53 inactivation in tumor cells [2]. In recent years, another member of the MDM family, MDM4 (also named MDMX) has come into the limelight and its function in the inactivation of $\mathrm{p} 53$ has been revealed by molecular and genetic approaches [1, 3]. In particular, MDM4 can negatively regulate p53-mediated cell cycle arrest, a role that distinguishes its function from that of MDM2, more related to the suppression of p53-mediated apoptotic response [1, 3-5]. However, others have reported on the antiproliferative and proapoptotic effect of MDM4 in the presence of wild-type TP53 under conditions such as stress $[6,7]$. Studies aimed at characterizing the human MDM4 (also named HDMX) status in human tumors have shown

\footnotetext{
N. Avenia

Perugia, Italy

H. J. Schünemann

Dept. of Epidemiology,

Regina Elena Cancer Institute,

Rome, Italy

L. Pistola $\cdot$ V. Ludovin

Lab of Medical Oncology,

Azienda Ospedaliera Perugia,

Perugia, Italy
}

Dept. of Surgery, University of Perugia, amplification of its locus in the presence of wild-type TP53 in breast cancers, glioblastoma, retinoblastoma, and softtissue sarcomas [8-11], confirming that it may contribute to p53 inactivation during tumorigenesis. The impact of human MDM4 on tumor properties as well as its behavior during tumor progression however still poorly explored.

In addition to the full-length (fl) protein, different splice variants of MDM4 have been described, two of which detected in human tumors. A short form named MDM4-S (a.k.a. HDMX-S) was identified first [12]. This variant derives from an alternative splicing that produces a truncated protein, containing essentially the p53-binding domain. It is a more potent inhibitor of p53 transcriptional activity than wt MDM4 and although present in both normal and tumor tissues, it is overexpressed specifically in tumor samples [11, 12]. Recently, our group has characterized another MDM4-splicing form, HDMX211 (hereafter named MDM4-211), in a thyroid tumor cell line and in some lung cancers [13]. This form derives from an aberrant splicing and produces a protein containing essentially the RING finger $\mathrm{COOH}$-terminus, where the MDM2 binding site resides. As a consequence, this variant is able to stabilize MDM2 protein levels, thus contributing to inactivation of $\mathrm{p} 53$. These data, while underlying the complexity of MDM4 expression in human tumors, strongly encourage studies on the comparative analysis of this protein and its derivative forms in human cancer.

Thyroid tumors represent over $90 \%$ of all endocrine cancers and are characterized by different genetic alterations, among which TP53 mutations are confined quite exclusively to the infrequent anaplastic and poorly differentiated histotype [14]. According to current models of human carcinogenesis that consider inactivation of the oncosuppressor $\mathrm{p} 53$, a common feature of almost all tumors, MDM2 amplification and/or overexpression have been reported in thyroid tumors too [15-17]. However, the overall frequency of MDM2 overexpression is only about $30 \%$, suggesting the existence of other pathways of p53 inactivation.

The aim of our study has been to analyze papillary thyroid carcinomas (PTC), the most frequent thyroid cancer, for the presence of MDM4 and its derivative forms to investigate alterations of these proteins in this tumor histotype and correlate them with histopathological features. In addition, we have analyzed MDM2 levels to assess the potential relationship between alterations of the two MDM family members. Our data have revealed the aberrant presence of variants MDM4-S and MDM4-211 messenger RNA (mRNA) in PTC as well as lack of correlation between MDM4 and MDM2 mRNA levels. Of note, levels of MDM4 mRNA were significantly downregulated in tumor samples in comparison to normal counterparts, and such downregulation appears significantly associated with tumor stage. 


\section{Materials and methods}

Tissue samples and patients

Fifty-seven papillary thyroid carcinomas and 57 matched normal thyroid tissue samples from the contra-lateral lobe (CTRL) of 57 patients were studied. In addition, to confirm statistical analyses, 26 papillary thyroid carcinomas and three normal thyroid tissues from independent individuals were analyzed. All specimens were obtained from patients undergoing surgery at the University of Perugia from 1997 to 2007. Before the surgical procedure, all patients signed informed consent forms for collection of fresh thyroid samples for genetic studies. All specimens were sampled from the primary tumor at the time of surgery, snap frozen, and stored at $-80^{\circ} \mathrm{C}$ until use. Tumors containing at least $70 \%$ of tumor cells based on the hematoxylin-eosin staining were selected. All normal thyroid tissue from the contra-lateral lobe were histopathologically analyzed for the presence of tumor. Available medical records of the patients were consulted to gain information about the clinical features of the disease and, when possible, the tumor stage was defined according to the sixth edition of the "American Joint Committee on Cancer" Cancer Staging Manual (American Joint Committee on Cancer 2002) based on pathological tumor-node-metastasis parameters and distinguishing patients in two groups: (1) patient age $<45$ years (stage I and II), (2) patient age $\geq 45$ years (stage I, II, III, IV).

The total of 83 papillary thyroid carcinomas included 55 of the classic variant, 20 of the follicular variant, five of other variants (tall cell, solid, diffuse sclerosing), and three showing the coexistence of PTC histology with areas of dedifferentiation. The mean period of follow-up was $45.8 \pm$ 30.4 months.

Genetic analysis of TP53 mutation, BRAF mutations and ret/PTC rearrangements

Analysis of the TP53 gene status was performed as follows: exons $5,6,7$, and 8 of $p 53$ gene were sequentially amplified by polymerase chain reaction (PCR) assay with the use of AmpliTaq Gold (Applied Biosystems) and following primer sets: exon 5 sense: TTCCTCTTCCTA CAGTACTC; exon 5 antisense: GCCCCAGCTGTTCAC; exon 6 sense: ACTGATTGCTCTTAG; exon 6 antisense: AGTTGCAAACCAGAC; exon 7 sense: AGTTGTGTT ATCTCCTAG; exon 7 antisense: CAAGTGGCTCCTGAC; exon 8 sense: TCCTATCCTGAGTAG; exon 8 antisense: GTCCTGCTTGCTTAC. Purified PCR products were sequenced in both directions with the use of the BigDye terminator Cycle sequencing Kit (version 1.1, Applied Biosystems) and an ABI Genetic Analyzer (Model 3130, Applied Biosystems). Sequence data were analyzed by means of SeqScape software (version 2.1, Applied Biosystems) followed by manual review.

Search for mutations of BRAF was conducted by singlestranded conformational polymorphism (SSCP) screening of real-time (RT)-PCR products of exons 15 , followed by sequencing, as previously described [18]. Screening for ret/ PTC 1 and ret/PTC 3 rearrangements was performed by RTPCR using primers spanning the breakpoints, as previously described [18].

Quantitative real-time PCR

Total RNA was extracted with Trizol ${ }^{\mathrm{TM}}$ (Invitrogen Corp., Carlsbad, CA, USA), according to the manufacturer's instructions. The expression of MDM2, MDM4, and MDM4-211 in tumors and matching normal thyroid samples was measured by quantitative real-time PCR (qRT-PCR), based on TaqMan methodology, using the ABI PRISM 7500 Sequence Detection System (Applied Biosystems, Foster City, CA) according to Giglio et al. [13]. Briefly, custom-designed TaqMan primers and probes (Applied Biosystems) specific for MDM4, MDM2, and MDM4-211 were used. Our results are expressed as relative units of target mRNA, referred to a sample called calibrator, chosen to represent $1 \times$ expression of the target gene. The calibrator used was the lowest value in the tissue collection under study. All analyzed samples express $n$-fold mRNA relative to the calibrator. Each sample mRNA was normalized relative to the $\beta$-actin mRNA. Predesigned TaqMan primers and probe (Applied Biosystems) for the housekeeping gene $\beta$-actin were used. Each sample was treated with deoxyribonuclease (DNase) I amplification grade (Invitrogen) and tested before and after DNase treatment.

Comparative analysis of flMDM4 and MDM4-S was driven by qRT-PCR according to Bartel et al., [11] using specific probes and SYBR Master mix (Applied Biosystems) with evaluation of dissociation curves. A MDM4-Sto-flMDM4 ratio of $>1.5$ was considered indicative of overexpressed MDM4-S.

\section{Western blot analysis}

Tumor and normal tissue pairs whose material was sufficient for western blot analysis were selected. All samples were homogenized with Tissue Lyser (Qiagen) and western blots were performed according to Giglio et al. [13]. Briefly, samples were lysed in radio-immunoprecipitation assay buffer $(50 \mathrm{mM}$ Tris-Cl pH 7.5, $150 \mathrm{mM} \mathrm{NaCl}, 1 \%$ Nonidet $\mathrm{P}-40,0.5 \% \mathrm{Na}$ desoxicholate, $0.1 \%$ sodium dodecyl sulfate (SDS), $1 \mathrm{mM}$ ethylenediaminetetraacetic acid) supplemented with a cocktail of protease inhibitors (Boehringer). Whole lysates were boiled in SDS Laemnli sample buffer, resolved by SDS-polyacrylamide gel elec- 
trophoresis on precast $10 \%$ gels (Invitrogen) and subsequently transferred to polyvinylidene fluoride membranes (Millipore). After protein transfer, membranes were blocked for $30 \mathrm{~min}$ with Tris-buffered saline containing $0.1 \%$ Tween-20 and 5\% nonfat dry milk and incubated with specific antibodies. The following primary antibodies were used: rabbit anti-MDM4 polyclonal antibody R1 (raised against full-length MDM4 protein) and (Bethyl Laboratories) mouse anti-MDM2 monoclonal antibody $2 \mathrm{~A} 10$ and monoclonal antibody Ab-1 (oncogene), mouse anti- $\alpha$ tubulin monoclonal antibody DM-1A (Sigma), and mouse $\alpha$-actin monoclonal antibody AC-40 (Sigma). MDM4 was probed with both Bethyl and R1 antibodies, giving similar results although, in comparison, the polyclonal antibody R1 was more sensitive. The homemade R1 antibody has been raised using the entire MDM4 protein. Experiments of epitope mapping by using MDM4-deletion mutants have shown that $\mathrm{R} 1$ does not recognize the $\mathrm{NH} 2$-terminus of MDM4. It is therefore unable to recognize the MDM4-S form.

\section{Statistical analysis}

Statistic analysis was carried out using the Analyze-it software for Microsoft Excel (Analyze-it Software, Ltd.). Spearman rank correlations and Kendall rank correlation (for measure of linear association) were used to evaluate the correlation between not-normally-distributed variables. To compare groups, we used the Wilcoxon Signed-Ranks test for related samples (57 tumors vs. matched thyroid normal tissues) and Mann-Whitney $U$ test for independent samples
(83 tumors vs. 60 normal thyroid samples). A probability of $p<0.05$ was considered as statistically significant. Intraindividual variation was calculated by utilizing the T-to-N ratio that derives from the ratio of corrected tumor (mRNA or protein) levels to corrected normal tissue levels of MDM4 and MDM2.

\section{Results}

Analysis of MDM4 and MDM2 levels in papillary thyroid tumors

A group of 57 thyroid tumors samples and 57 matched normal thyroid tissues from the CTRL were analyzed. All tumors were of papillary histotype (PTC). The mean age of the studied population was $49.4 \pm 16.81$ (SD); the female percentage was $72 \%$. The histopathological features of the 57 tumors are shown in Table 1 . Tumor stage was defined according to the sixth edition of the "American Joint Committee on Cancer."

All samples were screened for the presence of TP53 mutations (limitedly to exons $5-8$ where $80 \%$ of mutations occur), BRAF mutations (by SSCP followed by sequence analysis), and for ret/PTC 1 and ret/PTC 3 rearrangements (by RT-PCR; Table 2). MDM4 and flMDM2 mRNA levels were evaluated by qRT-PCR, using specific primers and probes. MDM4 primers were chosen in a region not present in the variant forms MDM4-S, HDMX-G [19] MDM4-211, XALT1, and XALT2 [20] to amplify specifically the fl
Table 1 Histopathological features of the 57 Papillary Thyroid Tumors (PTC)

\begin{tabular}{lccc}
\hline & Yes, $n(\%)$ & No, $n(\%)$ & ND, $n(\%)$ \\
\hline BRAF mutation & $35(61)$ & $20(35)$ & $2(3)$ \\
Ret/PTC rearrangement & $5(9)$ & $50(88)$ & $2(3)$ \\
TP53 mutation & $0(0)$ & $57(100)$ & 0 \\
Extra-thyroidal invasion & $15(26)$ & $41(72)$ & $1(2)$ \\
Multifocality & $24(42)$ & $32(56)$ & $1(2)$ \\
Nodal metastasis & $24(42)$ & $33(58)$ & 0 \\
Distant metastasis & $6(10)$ & $35(62)$ & $5(28)$ \\
Tumor stage of patients $<45$ years $(n=20)$ & & $5(25)$ \\
I & $15(75)$ & $15(75)$ & $11(30)$ \\
II & $0(0)$ & & $11(30)$ \\
Tumor stage of patients $\geq 45$ years $(n=37)$ & $18(48)$ & $11(30)$ \\
I & $8(22)$ & $24(65)$ & $11(30)$ \\
II & $2(5)$ & $23(62)$ & 0 \\
III & $3(8)$ & $13(35)$ & 0 \\
IV & $13(35)$ & $19(33)$ & 0 \\
Histology & $38(67)$ & $44(77)$ & 0 \\
Classic & $13(23)$ & $53(93)$ & $55(97)$ \\
Follicular & $4(7)$ & & \\
Other & $2(3)$ & & \\
Dedifferentiated & & & \\
& & &
\end{tabular}




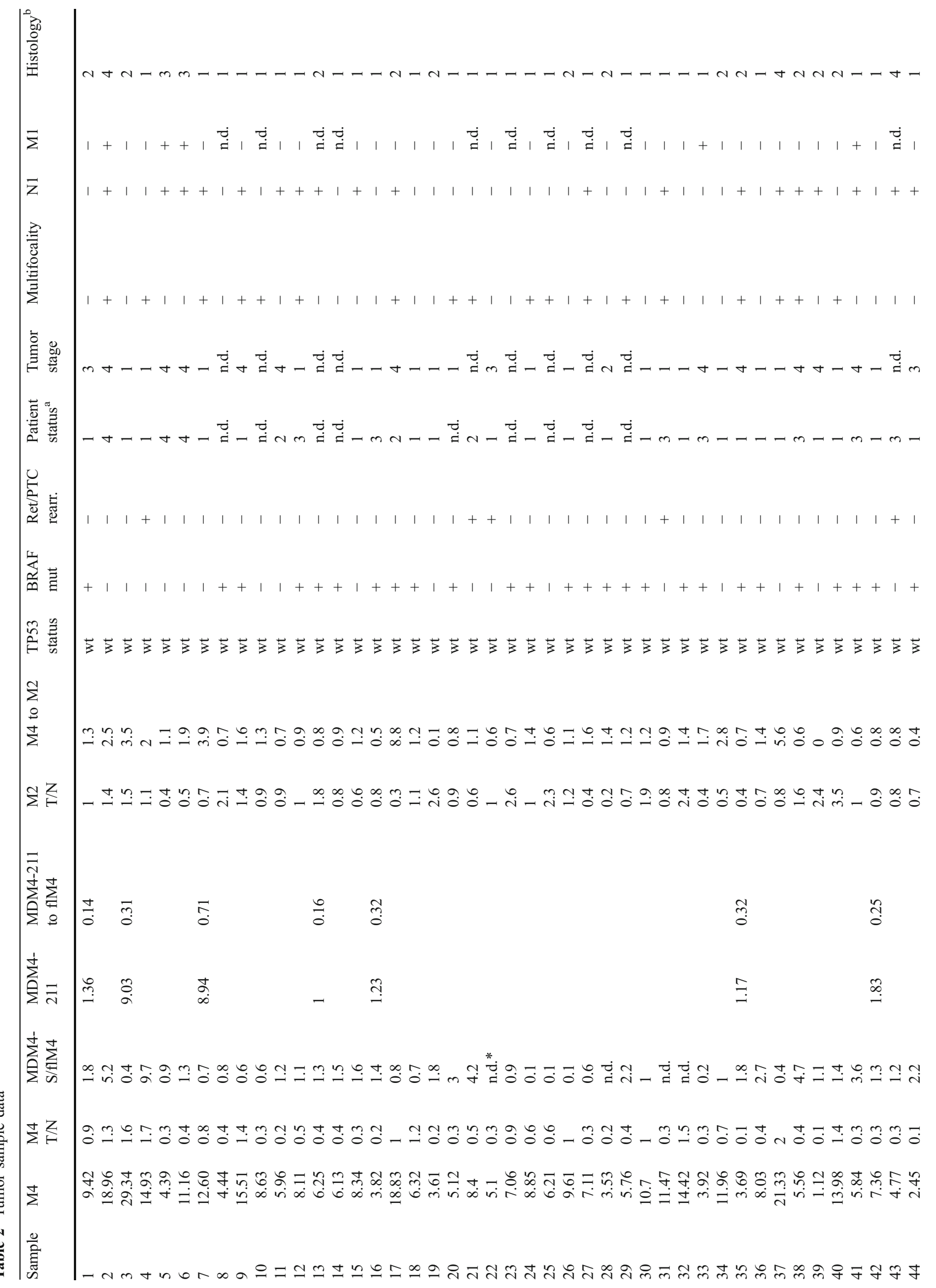




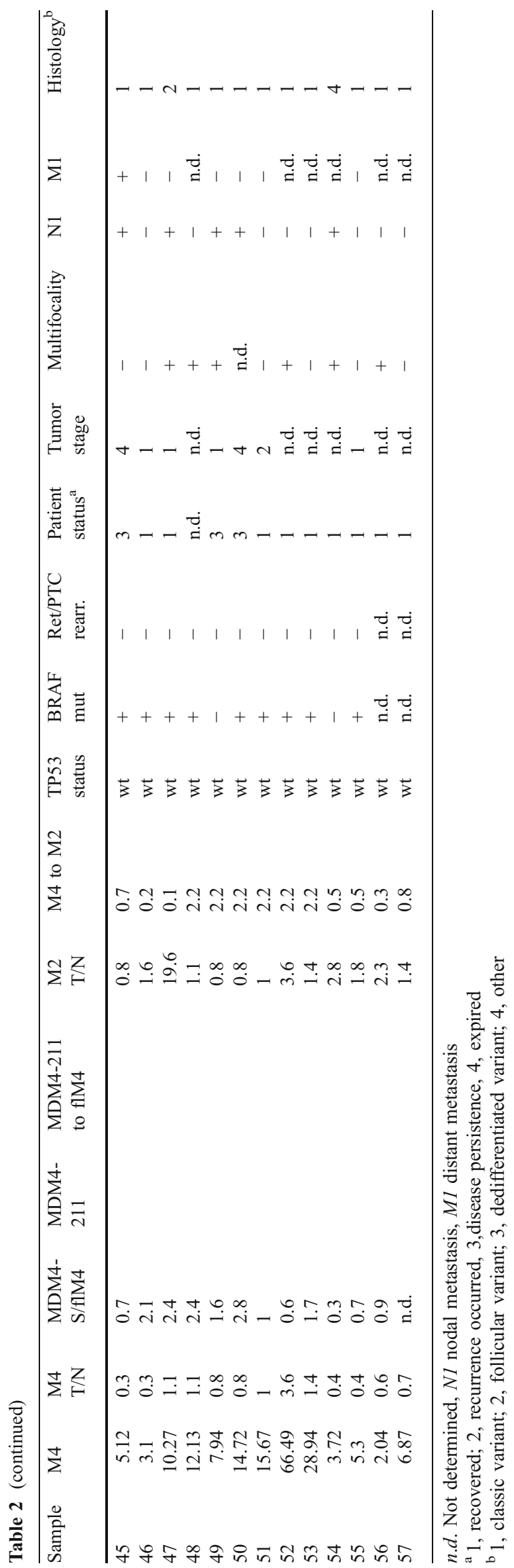

product. Similarly, the chosen MDM2 primers do not amplify the MDM2-A, MDM2-B, MDM2-C, MDM2-D, and MDM2-E alternative spliced forms and most of the aberrantly spliced forms [21].

MDM4 and MDM2 mRNA levels in normal tissues followed a normal distribution according to Kurtosis analysis (Fig. 1a,b). On the contrary, MDM4 and MDM2 values in tumor samples were not normally distributed $(p<$ 0.0001; Fig. 1c,d). Comparative analysis by Wilcoxon signed-ranks test for paired samples revealed a highly significant difference in the distribution of MDM4 values between tumor and CTRL groups (Wilcoxon's $W=1,385$, $p<0.0001$; Fig. 1e). Conversely, comparison of MDM2 values did not show significant difference $(p=0.61$; Fig. 1f), in spite of the presence of four upper outliers in the tumor sample set. There was no correlation between MDM4 and MDM2 mRNA tumor levels indicating that the alterations of the two transcripts are independent from one another (data not shown).

To confirm the decrease of MDM4 mRNA levels in PTC in comparison to CTRL, we analyzed these levels in an additional 26 independent PTC and three normal thyroid samples. Comparison of tumor and CTRL groups still resulted in a highly significant correlation (Mann-Whitney for independent samples $U=634, p<0.0001$ ) and confirmed previous results of a strong downregulation of MDM4 expression in tumor samples. Indeed, study of prediction by receive operator characteristics curves (ROC curves) indicated that the use of MDM4 mRNA levels as potential diagnostic test has a highly significant ability to discriminate between normal and tumor tissues (area under the ROC 0.84, $p<0.0001$ Fig. 2).

To ascertain whether the decrease of MDM4 mRNA in tumor samples results in decreased protein levels, we analyzed whole-cell extracts from 20 available pairs of tumors and CTRLs (Fig. 3). In 16 out of 20 pairs, the tumor-toCTRL ratio of densitometric values of the MDM4 protein levels correlated significantly with the observed ratio of the mRNA levels (Kendall tau $=0.7, p=0.0002$ ). Interestingly, western blot confirmed the decrease of MDM4 mRNA in tumor relative to normal tissue in seven samples, reinforcing previous observations. Notably, in three samples $(44,27$, and 33 ), we observed an increase of MDM4 proteins in tumor compared to CTRL despite equal or even lower levels of corresponding tumor mRNA relative to CTRL, indicating MDM4 protein stabilization independent of transcription. Western blot analysis revealed also the presence of an additional species of about $58 \mathrm{kDa}$ in some tumor samples (Fig. 3, arrows). This band was recognized also by a mix of three monoclonal antibodies to MDM4 (6B1A, 11F4D, and $12 \mathrm{G} 11 \mathrm{G}$, data not shown), suggesting it may be a MDM4 variant. However, its size does not correspond to any of the MDM4 variant forms described so far. 
Fig. 1 a-d Frequency histograms showing the distribution of the mRNA levels of the indicated genes in the indicated sample groups. Relative units of target mRNA are referred to a sample called calibrator, chosen to represent $1 \times$ expression of the target gene. Each sample mRNA was normalized relative to the $\beta$-actin mRNA. The superimposed line shows the normal distribution curve. For each distribution, the mean $(M) \pm$ standard deviation $(S D)$ is shown. e, f Comparison of MDM4 and MDM2 levels, respectively, between control and tumor samples. Each plot shows graphically the central location and scatter-dispersion of the values of each group: the line series shows parametric statistics (mean and confidence interval of mean) while the notched box and whiskers show nonparametric statistics (median, confidence interval of median, and interquartile range). Crosses and circles indicate possible outliers, between 1.5 and 3 interquartile range and over 3 interquartile range, respectively. $p$ value was calculated according to Wilcoxon signed-ranks test
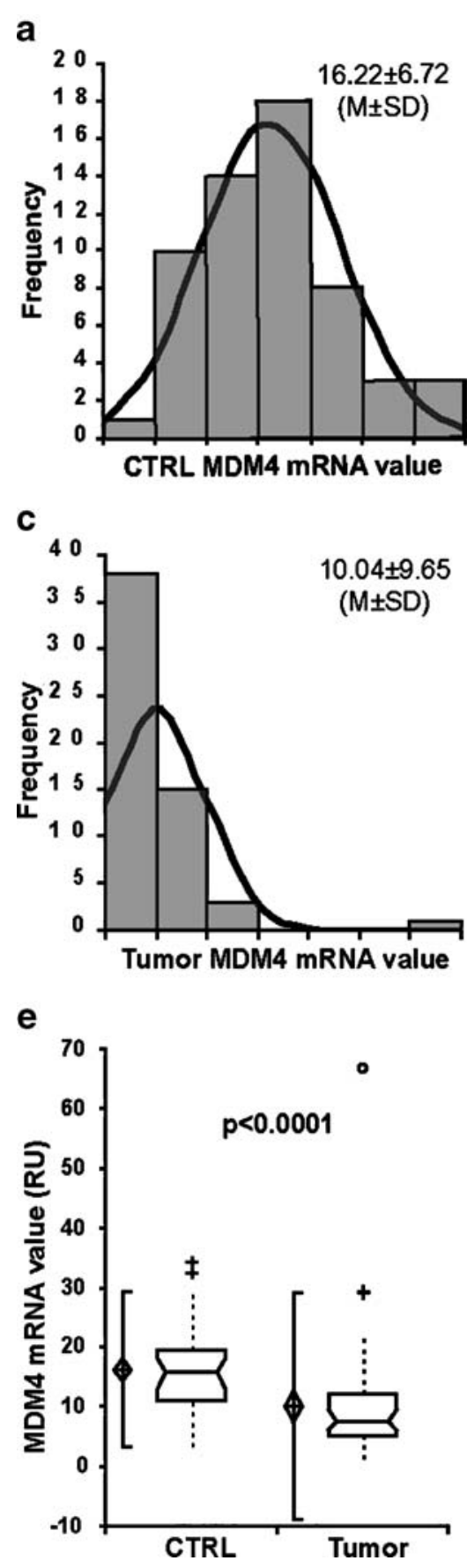

\section{b}

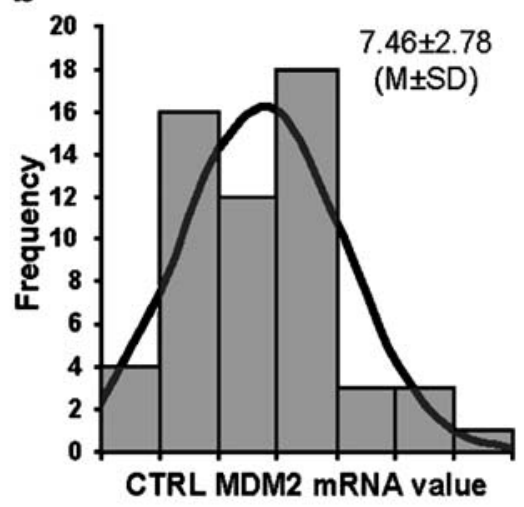

d
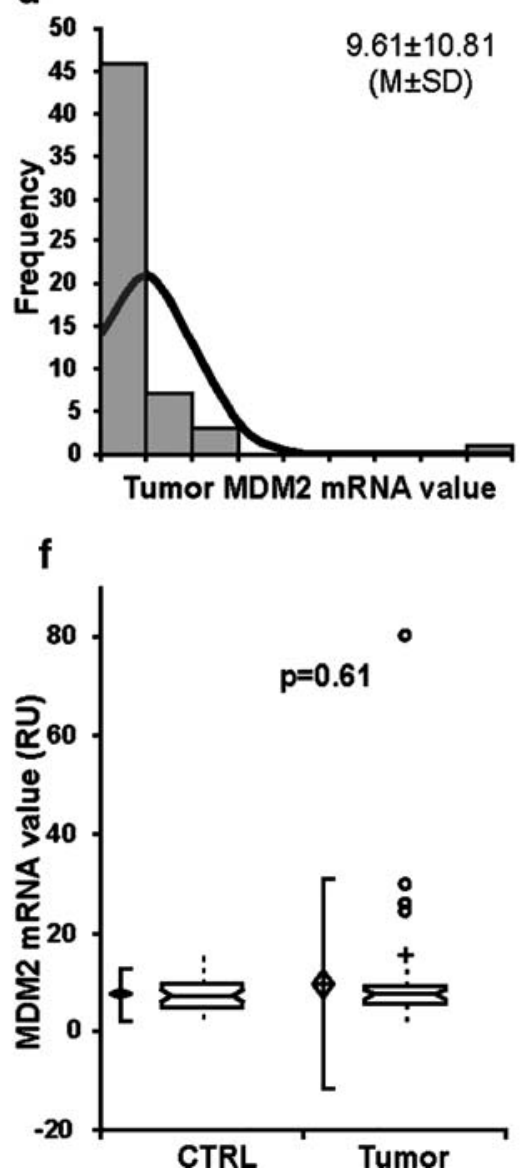

Further, western blot revealed frequent overexpression of the MDM2 protein in tumors that did not show overexpression of the mRNA (Fig. 3, samples marked by section signs).

Analysis of intraindividual variation of MDM4 and MDM2 levels

Previous data were obtained by comparing tumors versus CTRLs. We then analyzed the intraindividual variation of MDM4 and MDM2 mRNA levels for each tumor (T) and its matched CTRL $(\mathrm{N})$ by calculating the relative ratio, $\mathrm{T}$ to $\mathrm{N}$. The range of MDM4 T-to-N ratios varied from 0.1 to 3.6 (Fig. 4a and Table 2). The majority of tumor samples showed a decrease of MDM4 levels compared to the corresponding CTRLs (29 out of 57 samples having a ratio of $<0.5$ ), confirming the previously observed decrease of tumor MDM4 expression at individual levels too. In other tumor histotypes, amplification of MDM4 gene and overexpression of the mRNA have been reported. In this PTC group, MDM4 overexpression (as defined by a T-to-N ratio of $\geq 5$ ) was not detectable; in only two cases, the ratio of $\mathrm{T}$ 


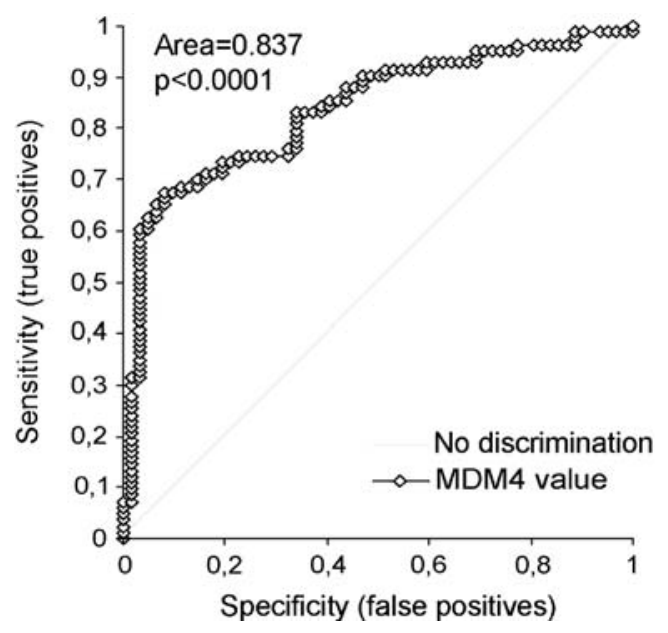

Fig. 2 Receive operator characteristics curves for MDM4 levels between normal and tumor samples, assessing variable MDM4 levels as diagnostic test. The plot shows sensitivity of the test against specificity. $p$ value was 0.0001 . For each decision threshold, the percentage of tumor cases correctly identified as such (true positives) against the percentage of normal cases incorrectly diagnosed as tumor (false positives) is shown

to $\mathrm{N}$ showed a value of $\geq 2$ ( 2 and 3.6 , respectively). On the contrary, comparison of MDM2 mRNA levels showed tumor values twofold higher than matching CTRL in 11 samples, among which one had a ratio of $>19$, suggesting an amplification event (Fig. 4b and Table 2). Moreover, only six samples $(10 \%)$ showed a ratio of $<0.5$, indicating that downregulation of MDM2 levels in PTC tumor is a rare event in comparison to that of MDM4. Notably, in two of these tumors, western blot analysis did not confirm the mRNA MDM2 decrease (Fig. 3; samples 35, 27).

Correlation of MDM4 and MDM2 with tumor histopathological features

We then investigated whether tumor MDM4 and MDM2 mRNA levels as well as the T-to-N ratio varied according to tumor properties by correlating them with the histopathological features of samples as reported in Table 1. First, we examined their association with BRAF mutations and ret/ PTC rearrangements, two hallmarks of thyroid carcinogenesis. BRAF mutation were detected in 35 out of 57 patients, ret/PTC rearrangements in 5 out of 57 (Table 2). All BRAF mutations but one involved a heterozygous $\mathrm{T}>\mathrm{A}$ transversion at nucleotide 1799, resulting in the prototypic valine-to-glutamic-acid substitution at position 600 $\left(\mathrm{BRAF}^{\mathrm{V} 600 \mathrm{E}}\right)$. In one case, the in-frame insertion at position 1796 of an additional codon, coding for Valine, was detected $\left(\mathrm{BRAF}^{\mathrm{V} 599 \text { Ins }}\right)$. This was shown to be a "gain-offunction" mutation in all super imposable to $\mathrm{BRAF}^{\mathrm{V} 600 \mathrm{E}}$ [22]. No association was found between MDM4 and MDM2 expression, BRAF mutations, and ret/PTC rearrangements suggesting that these oncogenic hits are independent events. We then analyzed the correlation between MDM4 or MDM2 expression with the other tumor histopathological features. This revealed association of MDM4 with multifocality of tumors: specifically, tumors presenting as multifocal show higher values than nonmultifocal (Spearman rank correlation $\mathrm{rs}=0.29, p=0.032$; Fig. 5a, Table 3). This association was not evident when we correlated multifocality with the MDM4 levels of matching normal tissues $(p=0.23$ ), indicating that this tumor property is not attributable to a patient predisposition given by individual MDM4 expression levels but rather it is a specific feature of tumor cells.

We also correlated MDM4 T-to-N ratio with tumor features. We observed an even stronger association between this parameter and multifocality (Spearman correlation coefficient $r s=0.32, p=0.01$, Table 3 ), confirming previous observations. Further, we found a significant correlation of MDM4 T-to-N ratio with tumor stage (Spearman correlation coefficient, $\mathrm{rs}=0.35$ for $n=41, p=0.02$; Fig. $5 \mathrm{~b}$ ); in particular, stage I tumors showed the highest ratio, indicating downregulation of MDM4 levels in late-stage tumors in comparison to early-stage ones (Table 3).

On the contrary, MDM2 levels as well as MDM2 T-to-N ratio did not show correlation with any histopathological parameter.

\section{Characterization of MDM4 variants in thyroid tumors}

We have analyzed the presence of the MDM4 variant forms, MDM4-S and MDM4-211, in the same sample group. So far, these are the only variant forms of MDM4 detected in human tumors. MDM4-S is a splicing variant present both in normal and tumor tissues; however, in tumor cells, MDM4-S is overexpressed and, as a consequence, the ratio MDM4-S to flMDM4 is $>1$. At first, we performed comparative analysis of flMDM4 and MDM4-S by qRT-PCR in CTRL samples. In agreement with the literature, we detected both transcripts in all samples with a ratio MDM4-S to fl-MDM4 ranging from 0.09 to 0.79 with only two samples showing values of 1.14 and 1.05 (data not shown). On the basis of these results, we considered overexpression as defined by a ratio of $\geq 1.5$ The same analysis in PTC detected MDM4-S in all but five samples; the resulting MDM4-S-to-flMDM4 ratio was $\geq 1.5$ in 20 out of 57 of tumors ( $35 \%$; Table 2$)$. The same percentage resulted from the analysis of the additional group of 26 tumors ( 9 out of 26), in agreement with other reports [11]. Statistical analysis did not reveal any significant association of MDM4S-to-flMDM4 ratio with the features described in Table 1.

The second variant form we investigated was MDM4211 (HDMX211), previously isolated from a thyroid tumor cell line [13]. MDM4-211 mRNA was present in 7 out of 57 original tumor samples and in 8 out of 26 additional 
Fig. 3 Western blot of WCE derived from 20 couples of tumor (T) and matching control (N). The values derive from the ratio of densitometric value of MDM4-actin, both at the protein and mRNA levels. Asterisks mark tumor samples expressing MDM4-211 mRNA; section signs mark tumor samples showing increased levels of MDM2 protein with no increased levels of the corresponding mRNA. Arrows mark a lower weight protein of about $58 \mathrm{kDa}$, recognized by different $\alpha$ MDM4 antibodies

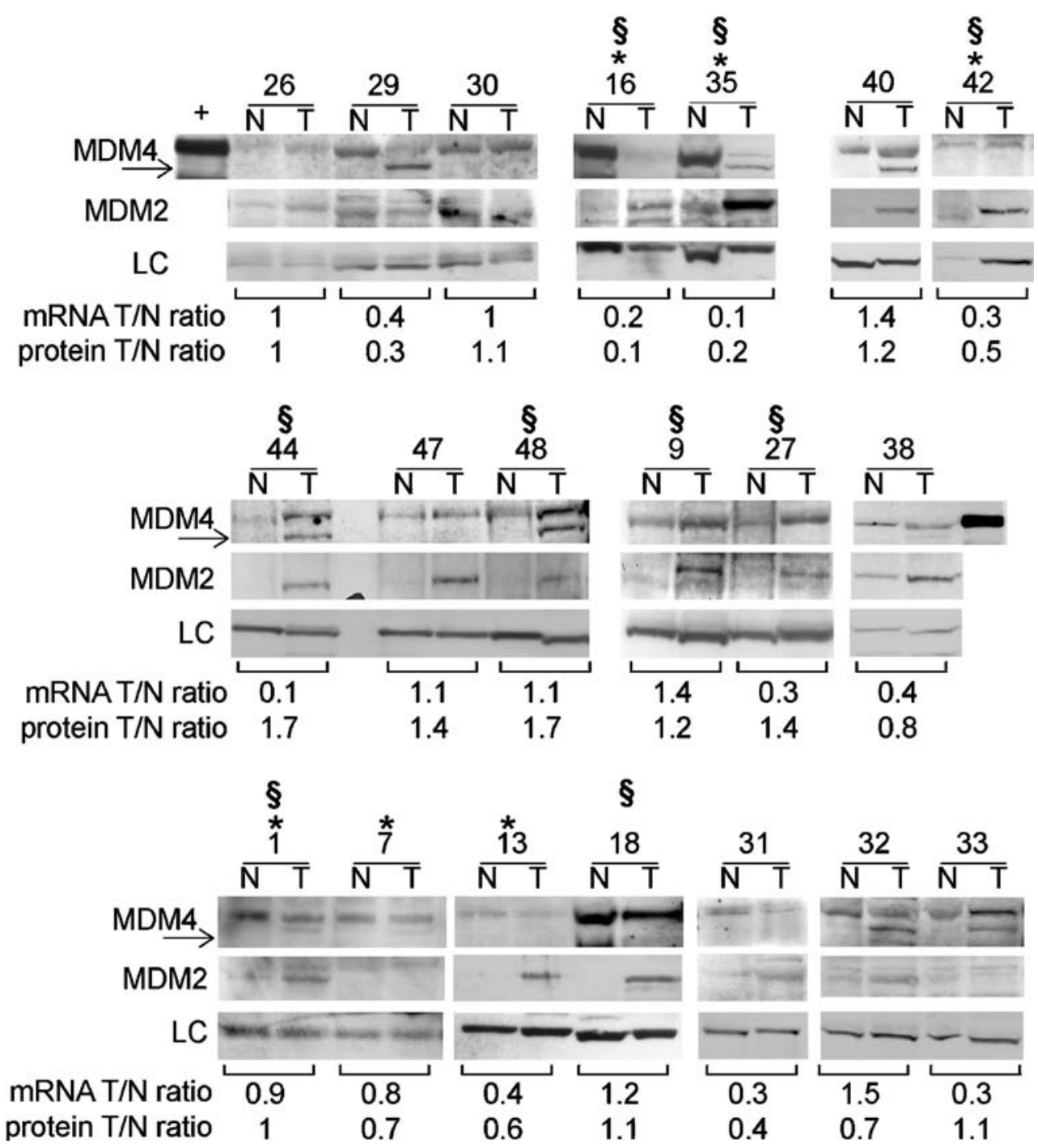

samples analyzed with an overall frequency of $18 \%$ (Table 2 and data not shown). Its mRNA was not detected in any of the CRTL samples, thus confirming its specific presence in tumor tissue. In agreement with our previous reports [13], western blot analysis of available tumor and CTRL samples confirmed enhanced levels of the oncoprotein MDM2 in tumors expressing MDM4-211 (Fig. 3, samples marked by *). In our tumor set, the presence of MDM4-211 did not
Fig. 4 a, b Frequency histograms showing the distribution of the T-to-N ratios derived from tumor-CTRL levels of the indicated genes. The superimposed line shows the normal distribution curve. For each distribution, the mean \pm standard deviation is shown a

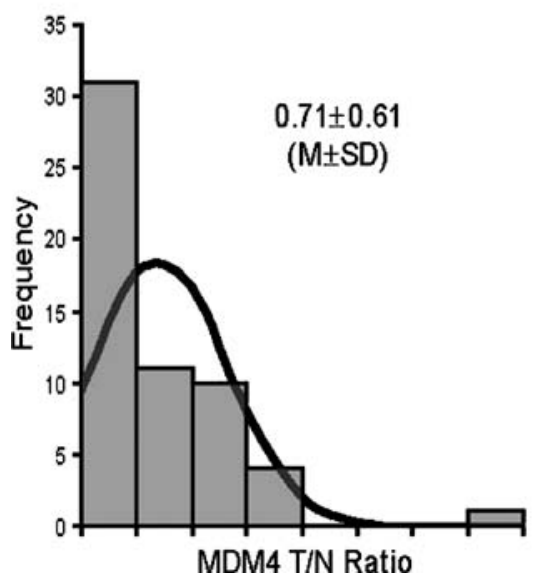

b

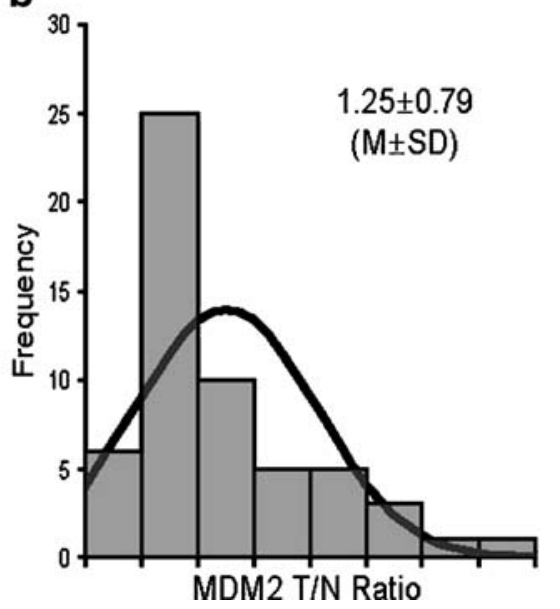




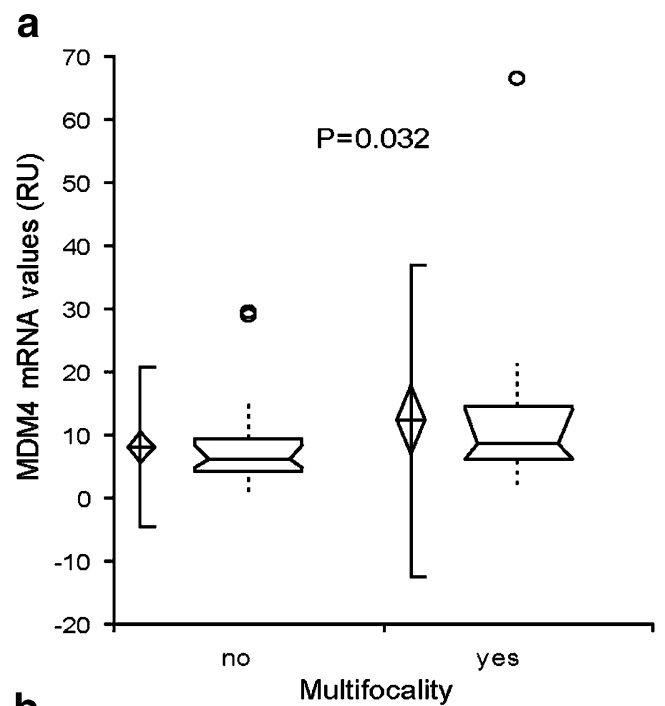

b

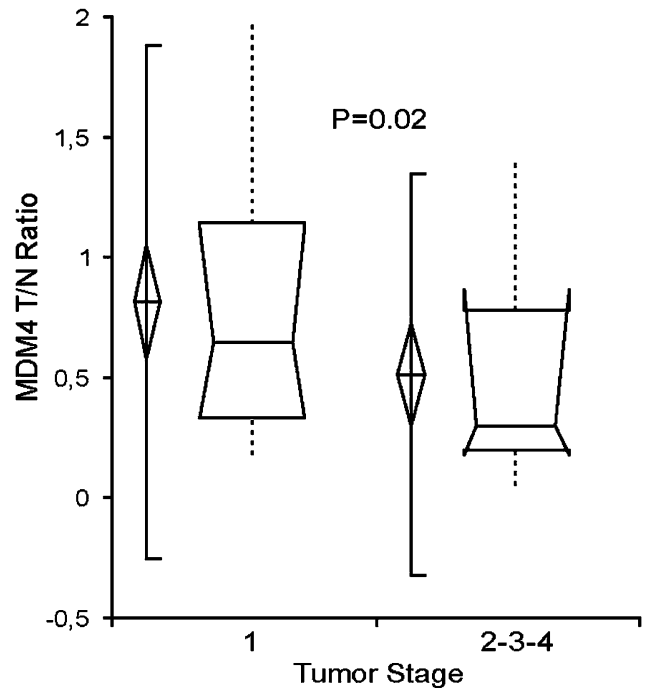

Fig. 5 a Vertical box-whisker plots showing MDM4 levels between nonmultifocal (no) and multifocal (yes) tumors. Each plot shows graphically the central location and scatter-dispersion of the values of each group: the line series shows parametric statistics (mean and confidence interval of mean) while the notched box and whiskers show nonparametric statistics (median, confidence interval of median, and interquartile range). Crosses and circles indicate possible outliers, between 1.5 and 3 interquartile range and over 3 interquartile range, respectively. $p$ value was calculated according to Spearman test. b Vertical box-whisker plots showing tumor-CTRL MDM4 mRNA levels in stage 1 compared to other stages of tumors. Each plot shows graphically the central location and scatter-dispersion of the values of each group: the line series shows parametric statistics (mean and confidence interval of mean) while the notched box and whiskers show nonparametric statistics (median, confidence interval of median, and interquartile range). Crosses and circles indicate possible outliers, between 1.5 and 3 interquartile range and over 3 interquartile range, respectively. $p$ value was calculated according to Spearman test

correlate with any of the properties described in Table 1 nor with the expression of the form MDM4-S, indicating that the presence of these two variants is an independent event.

Some authors have hypothesized that the presence of MDM4 variants may correlate with lower level of flMDM4
[11]. We therefore assessed the presence and expression of MDM4-S and MDM4-211 in correlation with fIMDM4 and with the MDM4 T-to-N ratio. We observed no significant correlation but rather a slight association between low levels of both MDM4-S and flMDM4 ( $p=0.056)$.

\section{Discussion}

In this study, we have analyzed the expression of MDM4 and MDM2 mRNAs in a group of 57 papillary thyroid tumors in comparison to matching normal tissues. We observed that MDM4 levels are significantly lower in tumor samples than in control. Specifically, tumor samples expressing levels of MDM4 lower than matching control are very frequent $(51 \%)$ and this decrease in expression appears to correlate significantly with higher tumor stages (stage I in comparison to the other), indicating that it occurs with tumor progression. These data led us to hypothesize that a robust presence of MDM4 might exert an antagonistic role in PTC evolution. To date, the majority of the literature has reported amplification of MDM4 in the presence of wt TP53 in different tumor histotypes [3], partly in contrast with our data. However, a different status of MDM4 in some cancer has also been reported. In a gene profiling analysis, MDM4 downregulation was observed in prostate tumors with poor prognosis in comparison to those with good prognosis in agreement with the hypothesis that MDM4 may be detrimental to tumor progression [23]. Moreover, two studies reported low levels of MDM4 protein in chronic myeloid leukemia tumor samples and in tumor cell lines [24, 25]. It would be interesting to know whether those cell lines have features resembling those of advanced-stage tumors. Some authors have suggested that low MDM4 expression levels may be correlated to the presence of a mutated TP53 [11, 25]. However, lack of detection of any of the most frequent mutation of TP53 gene in this PTC group does not support this hypothesis, at least in this type of cancer. Similarly, in the prestate tumor

Table 3 Summary of significant correlations of MDM4 and MDM4 $\mathrm{T}-\mathrm{N}$ values with histopathological features

\begin{tabular}{|c|c|c|c|c|}
\hline & \multicolumn{2}{|c|}{ Multifocality } & \multicolumn{2}{|l|}{ Tumor Stage } \\
\hline & $\begin{array}{l}\text { Presence } \\
n=24\end{array}$ & $\begin{array}{l}\text { Absence } \\
n=32\end{array}$ & $\begin{array}{l}\mathrm{I} \\
n=23\end{array}$ & $\begin{array}{l}\text { II-IV } \\
n=18\end{array}$ \\
\hline \multicolumn{5}{|l|}{ MDM4 T-N } \\
\hline Mean \pm SD & $0.9 \pm 0.76$ & $0.56 \pm 0.43$ & $0.81 \pm 0.54$ & $0.51 \pm 0.43$ \\
\hline Median \pm IQR & $0.63 \pm 0.83$ & $0.38 \pm 0.6$ & $0.65 \pm 0.81$ & $0.3 \pm 0.58$ \\
\hline \multicolumn{5}{|l|}{ MDM4 } \\
\hline Mean \pm SD & $12.4 \pm 12.6$ & $8.1 \pm 6.4$ & $10.28 \pm 5.9$ & $8.38 \pm 5.9$ \\
\hline $\operatorname{Median} \pm \mathrm{IQR}$ & $8.74 \pm 8.7$ & $6.19 \pm 5.1$ & $8.85 \pm 5.44$ & $5.7 \pm 8.0$ \\
\hline
\end{tabular}


study TP53 was found to be wild type by sequencing (A. Farsetti, personal communication).

These data counteract the holistic view of MDM4 as a p53 inactivation tool and suggest that its function may be more complex than previously considered, at least in some tissues. Thus far, molecular studies indicating MDM4 functions different from negative regulation of 53 have provided evidence that MDM4 may display antiproliferative or proapoptotic activities, under stress conditions $[6$, 7]. Further, a recent work has shown for MDM4 a role in suppressing tumorigenesis through the control of bipolar mitosis [26]. Whether these mechanisms occur in PTC remain to be elucidated.

In the analysis of tumor MDM4 expression levels, we found that high levels of MDM4 are associated with multifocal tumors. Different hypothesis have been postulated to explain the origin of these tumors. Certainly, multifocality is related to the high proliferative potential of thyrocites. MDM4 has been reported as inhibitor of p53mediated growth arrest [1]; thus, it might be hypothesized that the presence of MDM4 may predispose to multifocality and tumor development, while a decrease in its expression may confer an advantage to tumor progression. Interestingly, one study has documented a statistically significant association of MDM4 amplification with low-grade astrocytomas, thus supporting the hypothesis that high levels of MDM4 may represent an early advantageous event for tumor development [27]. Further, the association between MDM4 levels and tumor multifocality is of particular clinical relevance. Indeed, the possibility of early diagnosis of tumor multifocality might allow discrimination between partial or total thyroid gland ablation. The extension of this type of analysis to a broader set of samples, including benign lesions, should help to confirm these findings and to investigate potential applications of this molecule in clinical practice.

In addition, we have observed with high frequency the aberrant presence of MDM4 alternatively spliced forms, whose oncogenic activity has been assessed so far only in vitro. In our analysis, the aberrant presence of these variants did not correlate with any of the histopathological features. In a study with primary sarcomas, the MDM4-S-toflMDM4 ratio correlated with enhanced cell proliferative potential and decreased patient survival [11]. The high incidence of patient survival in PTC (only three deaths in our group, Table 2) does not allow to confirm this association. For MDM4-211, this is the first frequency report in a large group of tumor samples and indicates that its presence is appreciable in PTC (18\%). Interestingly, these MDM4 variants forms show in vitro an even stronger inhibitory activity toward p53 than the full-length protein. Their presence may concur to modify the activity of flMDM4, making more complex the assessment of its function in tumor cells. It has to be mentioned that in many PTC samples we observed the presence of a MDM4 minor form whose nature is unclear, supporting the hypothesis that MDM4 variants may concur to MDM4 oncogenic functions.

Conversely, MDM2 mRNA levels were increased in majority of tumor samples. In particular, we observed with high frequency enhanced protein levels in tumor samples that do not show enhanced mRNA levels. This is in accord with previous reports and confirms that in thyroid tumors MDM2 overexpression takes place frequently by mechanisms other than enhanced transcription [28]. At least in some of these samples, the simultaneous presence of MDM4-211 variant may cause such upregulation. These data further confirm the general view of the antagonistic role of MDM2 toward the oncosuppressor p53. Finally, in this study, we did not find any correlation between MDM2 and MDM4 mRNA levels, suggesting that the alterations we observed occur independently from one another.

Overall, our results reveal that MDM4 alterations, particularly the presence of its variant forms with p53inhibitory properties, are present at high frequency in PTC confirming the general model of different ways of p53 inactivation in human tumors. However, our data represent the first statistical report of a strongly significant downregulation of flMDM4 expression in cancer and particularly in advanced-stage tumors, suggesting that this protein may play additional roles besides p53 inactivation at least in PTC. In view of the suggested strategies for abrogation of MDM4 in human tumors [1, 3], our findings counsel acquisition of more information on the molecular functions of this protein in different human tumors in order to carefully evaluate the application of such therapies.

Acknowledgements We are grateful to Dr. Silvia Bacchetti and Dr. Silvia Soddu for helpful discussion. A.P and S.G. equally contributed to this work.

Funding This work has been supported by grants from the Italian Association for Cancer Research and Ministero dell'Università e della Ricerca. F. Mancini is a recipient of a Fondazione Italiana per la Ricerca sul Cancro fellowship.

Open Access This article is distributed under the terms of the Creative Commons Attribution Noncommercial License which permits any noncommercial use, distribution, and reproduction in any medium, provided the original author(s) and source are credited.

\section{References}

1. Marine J-C, Francoz S, Maetens M, Wahl G, Toledo F, Lozano G (2006) Keeping p53 in check: essential and synergistic functions of Mdm2 and Mdm4. Cell Death Differ 13:927-934 
2. Momand J, Jung D, Wilczynski S, Niland J (1998) The MDM2 gene amplification database. Nucleic Acids Res 26:3453-3459

3. Marine J-C, Dyer MA, Jochemsen AG (2007) MDMX; from bench to bedside. J Cell Sci 120:371-8

4. Shmueli A, Oren M (2007) Mdm2: p53's lifesaver? Mol Cell 25:794-796

5. Rinaldo C, Prodosmo A, Mancini F, Iacovelli S, Sacchi A, Moretti F, Soddu S (2007) MDM2-regulated degradation of HIPK2 prevents p53Ser46 phosphorylation and DNA damage-induced apoptosis. Molecular Cell 25:739-750

6. Mancini F, Gentiletti F, D'Angelo M, Giglio S, Nanni S, D'Angelo C, Farsetti A, Citro G, Sacchi A, Pontecorvi A, Moretti F (2004) MDM4 (MDMX) overexpression enhances stabilization of stress-induced p53 and promotes apoptosis. J Biol Chem 279:8169 8180

7. Steinman HA, Sluss HK, Sands AT, Pihan G, Jones SN (2004) Absence of p21 partially rescues Mdm4 loss and uncovers an antiproliferative effect of Mdm4 on cell growth. Oncogene 23:303-306

8. Laurie NA, Donovan SL, Shih CS, Zhang J, Mills N, Fuller C, Teunisse A, Lam S, Ramos Y, Mohan A, Johnson D, Wilson M, Rodriguez-Galindo C, Quarto M, Francoz S, Mendrysa SM, Guy RK, Marine JC, Jochemsen AG, Dyer MA (2006) Inactivation of the p53 pathway in retinoblastoma. Nature 444:6166

9. Riemenschneider MJ, Buschges R, Wolter M, Reifenberger J, Bostrom J, Kraus JA, Schlegel U, Reifenberger GH (1999) Amplification and overexpression of the MDM4 (MDMX) gene from 1q32 in a subset of malignant gliomas without TP53 mutation or MDM2 amplification. Cancer Res 59:6091-6096

10. Danovi D, Meulmeester E, Pasini D, Migliorini D, Capra M, Frenk R, de Graaf P, Francoz S, Gasparini P, Gobbi A, Helin K, Pelicci PG, Joechemsen AG, Marine JC (2004) Amplification of Mdmx (or Mdm4) directly contributes to tumor formation by inhibiting p53 tumor suppressor activity. Mol Cell Biol 24:58355843

11. Bartel F, Schulz J, Bohnke A, Blumke K, Kappler M, Bache M, Schimdt H, Wurl P, Taubert H, Hauptmann S (2005) Significance of HDMX-S (or MDM4) mRNA splice variant overexpression and HDMX gene amplification on primary soft tissue sarcoma prognosis. Int J Cancer 117:469-475

12. Rallapalli R, Strachan G, Cho B, Mercer WE, Hall DJ (1999) A novel MDMX transcript expressed in a variety of transformed cell lines encodes a truncated protein with potent p53 repressive activity. J Biol Chem 274:8299-8308

13. Giglio S, Mancini F, Gentiletti F, Sparaco G, Felicioni L, Barassi F, Martella C, Prodosmo A, Iacovelli S, Buttitta F, Farsetti A, Soddu S, Marchetti A, Sacchi A, Pontecorvi A, Moretti F (2005) Identification of an aberrantly spliced form of HDMX in human tumors: a new mechanism for HDM2 stabilization. Cancer Res 65:9687-9694

14. Moretti F, Nanni S, Pontecorvi AA (2000) Molecular pathogenesis of thyroid nodules and cancer. BaillieÁre's Clin Endocrinol Metab 14:517-539

15. Zou M, Shi Y, Al-Sedairy S, Hussain S, Farid NR (1995) The expression of the MDM2 gene, a p53 binding protein in thyroid carcinogenesis. Cancer 76:314-318
16. Jennings $T$, Bratslavsky $G$, Gerasimov $G$ (1995) Nuclear accumulation of MDM2 protein in well-differentiated papillary thyroid carcinomas. Exp Mol Path 62:199-206

17. Horie S, Maeta H, Endo K, Ueta T, Takashima K, Terada T (2001) Overexpression of p53 protein and MDM2 in papillary carcinomas of the thyroid: correlations with clinicopathologic features. Path Intern 51:11-15

18. Puxeddu E, Moretti S, Elisei R, Romei C, Pascucci R, Martinelli M, Marino C, Avenia N, Rossi ED, Fadda G, Cavaliere A, Ribacchi R, Falomi A, Pontecorvi A, Pacini F, Pinchera A, Santeusanio F (2004) BRAF(V599E) mutation is the leading genetic event in adult sporadic papillary thyroid carcinomas. J Clin Endocrinol Metab 89:2414-2420

19. de Graaf P, Little NA, Ramos YFM, Meulmeester E, Letteboer SJF, Jochemsen AG (2003) Hdmx protein stability is regulated by the ubiquitin ligase activity of Mdm2. J Biol Chem 278:3831538324

20. Chandler DS, Singh RK, Caldwell LC, Bitler JL, Lozano G (2006) Genotoxic stress induces coordinately regulated alternative splicing of the p53 modulators MDM2 and MDM4. Cancer Res 66:9502-9508

21. Bartel F, Taubert H, Harris LC (2002) Alternative and aberrant splicing of MDM2 mRNA in human cancer. Cancer Cell 2:9-15

22. Moretti S, Macchiarulo A, De Falco V, Avenia N, Barbi F, Carta C, Cavaliere A, Melillo RM, Passeri L, Santeusanio F, Tartaglia M, Santoro M, Puxeddu E (2006) Biochemical and molecular characterization of the novel BRAF ${ }^{\text {V599Ins }}$ mutation recently detected in a classic papillary thyroid carcinoma. Oncogene 25:4235-4240

23. Nanni S, Priolo C, Grasselli A, D'Eletto M, Merola R, Moretti F, Gallucci M, De Carli P, Sentinelli S, Cianciulli AM, Mottolese M, Carlini P, Arcelli D, Helmer-Citterich M, Gaetano C, Loda M, Bachetti S, Sacchi AFarsetti A (2006) Epithelial-restricted gene profile of primary cultures from human prostate tumors: a molecular approach to predict clinical behavior of prostate cancer. Mol Cancer Res 4:79-92

24. Pizzatti L, Ayres Sa L, Menezes de Souza J, Pisch PM, Abdelhay E (2006) Altered protein profile in chronic myeloid leukemia chronic phase identified by a comparative proteomic study. Biochim Biophys Acta 1764:929-942

25. Ramos YF, Stad R, Attema J, Peltenburg LT, van der Eb AJ, Jochemsen AG (2001) Aberrant expression of HDMX proteins in tumor cells correlates with wild-type p53. Cancer Res 61:1839-1842

26. Matijasevic Z, Steinman HA, Hoover K, Jones SN (2008) MDMX promotes bipolar mitosis to suppress transformation and tumorigenesis in p53-deficient cell and mice. Mol Cell Biol 28(4):12651273

27. Arjona D, Bello MJ, Alonso E, Isla A, De Campos JM, Vaquero J, Sarasa JL, Gutierrez M, Rey JA (2005) Real-time quantitative PCR analysis of regions involved in gene amplification reveals gene overdose in low-grade astrocytic gliomas. Diagn Mol Pathol 14:224-229

28. Evans SC, Viswanathan M, Grier JD, Narayana M, El-Naggar A, Lozano G (2001) An alternatively spliced HDM2 product increases p53 activity by inhibiting HDM2. Oncogene 20:4041-4049 